\title{
O USO DE ÁlCOOL E TABACO ENTRE ACADÊMICOS DE UMA UNIVERSIDADE DO SUL DO BRASIL
}

\section{The use of alcohol and tobacco among students of a university in Southern Brazil \\ El uso del alcohol y tabaco en académicos de una universidad del Sur de Brasil}

\section{Lucimare Ferraz}

Universidade Comunitária da Região de Chapecó - Unochapeco - Chapecó (SC) - Brasil

Shayane Luiza Rebelatto

Universidade Comunitária da Região de Chapecó - Unochapeco - Chapecó (SC) - Brasil

Greice Cristine Schneider

Universidade Comunitária da Região de Chapecó - Unochapeco - Chapecó (SC) - Brasil

Vinicius Anzolin

Universidade Federal do Rio Grande - FURG - Rio Grande (RS) - Brasil

\section{RESUMO}

Objetivo: Conhecer a prevalência do consumo de álcool e tabaco entre estudantes de uma universidade do sul do Brasil. Métodos: O estudo tem caráter exploratório-descritivo, com delineamento transversal, e ocorreu de agosto de 2012 a junho de 2013. A pesquisa foi realizada por meio de um questionário estruturado (com questões sobre o uso de álcool e tabaco) autopreenchido por 284 acadêmicos e os resultados foram analisados por técnicas descritivas, frequências absolutas e relativas, e estatísticas com teste qui-quadrado para variáveis categóricas dicotômicas e Kruskal-Wallis para variáveis contínuas. Resultados: Dos universitários participantes $242(85,2 \%)$ já consumiram álcool e $53(18,7 \%)$ já fizeram uso de tabaco em algum momento na vida. Em relação ao consumo das substâncias e o gênero dos universitários, não houve diferença em relação ao consumo de álcool ( $\mathrm{p}=0,857)$. Contudo, em relação ao tabaco, evidencia-se que os homens consomem mais que as mulheres $(\mathrm{p}=0,001)$. Conclusão: $\mathrm{O}$ estudo revela que a prevalência do consumo de álcool nessa população universitária é alta, tornando-se necessárias investigações mais abrangentes sobre os aspectos envolvidos no consumo de álcool e tabaco por esse público.

Descritores: Uso de Tabaco; Bebidas Alcoólicas; Estudantes; Prevalência.

\section{ABSTRACT}

Objective: To identify the prevalence of alcohol and tobacco consumption among undergraduate students of a university in Southern Brazil. Method: This is an exploratory-descriptive study, with a cross-sectional design, held from August 2012 to June 2013. The research was carried out by means of a structured questionnaire (with questions about alcohol and tobacco use), self-answered by 284 university students, and the results were analyzed through descriptive techniques, absolute and relative frequencies, and statistical tests, chi-square for dichotomous categorical variables and Kruskal-Wallis for continuous variables. Results: Of the undergraduates who participated in this study, $242(85.2 \%)$ have already consumed alcohol and 53 students (18.7\%) have already used tobacco at some point in their lives. Regarding the consumption of substances and the gender of the undergraduates, there was no difference in alcohol consumption ( $p=0.857)$. However, as for tobacco, men's consumption was evidenced to be higher than women's $(p=0.001)$. Conclusion: The study reveals that the prevalence of alcohol consumption among the university population is high, thus demanding broader investigations about the aspects involved in alcohol and tobacco consumption among this population.

Descriptors: Tobacco Use; Alcoholic beverages; Students; Prevalence.

\section{RESUMEN}

Objetivo: Conocer la prevalencia del consumo de alcohol y tabaco en estudiantes de una universidad del Sur de Brasil. Métodos: El estudio tiene el carácter exploratorio-descriptivo con delineamiento transversal y se dio entre agosto de 2012 y junio de 2013. Se realizó la 
investigación a través de un cuestionario estructurado (con preguntas sobre el uso del alcohol y tabaco) de autorelleno en 284 académicos y los resultados fueron analizados a través de técnicas descriptivas, frecuencias absolutas y relativas y estadistica con la prueba de Chicuadrado para las variables categóricas dicotómicas y la de Kruskal-Wallis para las variables continuas. Resultados: Entre los universitarios participantes $242(85,2 \%)$ ya han consumido el alcohol y $53(18,7 \%)$ ya usaron el tabaco en algún momento de su vida. No hubo diferencia respecto el consumo de alcohol y el género de los universitarios ( $p=0,857)$. Sin embargo, respecto al tabaco, se evidenció que los hombres los consumen más que las mujeres $(p=0,001)$. Conclusión: El estudio revela que la prevalencia del consumo de alcohol en esa población universitaria es alta con la necesidad de investigaciones más amplias sobre los aspectos del consumo de alcohol y tabaco por ese público.

Descriptores: Uso de Tabaco; Bebidas Alcohólicas; Estudiantes; Prevalencia.

\section{INTRODUÇÃO}

Ingressar no meio acadêmico representa uma nova etapa de vida para os jovens estudantes. Ser um universitário significa ter liberdade e controle sobre sua vida para a maioria deles. Todavia esse período na vida é considerado de vulnerabilidade às situações de risco social, frequentemente associada com o consumo excessivo de substâncias psicoativas, em destaque o álcool ${ }^{(1)}$.

A passagem da adolescência para a vida adulta implica em movimentos de ruptura, que podem ser compreendidos pelos jovens como atitudes de transgresão e de novas experiências. Dentre as novas vivências, descata-se o uso de substâncias psicoativas. Comumente, o primeiro contato do indivíduo com substâncias psicoativas ocorre na juventude, e essa experimentação pode se transformar em ocasional, moderada, e até abusiva ${ }^{(2)}$.

Entre as sustâncias psicoativas de uso dos universitários, de acordo com a Organização Mudial da Saúde (OMS), do uso controlado ao problemático, o álcool é a substância mais consumida. Trata-se de um problema de saúde pública, uma vez que o uso do álcool é um dos fatores de risco de maior impacto para a morbidade e mortalidade, estando relacionado a 3,3 milhões de mortes a cada ano. Assim, aproximadamente $6 \%$ de todas as mortes no mundo são atribuídas total ou parcialmente ao álcool. É possível reconhecer inúmeros prejuízos decorrentes do uso de álcool, tais como: violência interpessoal, acidentes de trânsito, relações sexuais sem proteção, neoplasias, pancreatite, cirrose hepática, entre outros ${ }^{(3)}$.

Outra sustância que vem tendo seu consumo aumentado entre os universitários é o tabaco, sendo fator causal para quase 50 diferentes doenças incapacitantes e fatais. Ele responde por $45 \%$ das mortes por infarto do miocárdio, $85 \%$ das mortes por doença pulmonar obstrutiva crônica (enfisema), $25 \%$ das mortes por doença cerebrovascular (derrames) e 30\% das mortes por câncer. Além disso, $90 \%$ dos casos de câncer de pulmão ocorrem em fumantes, elevando também o risco de desenvolverem a tuberculose. A cada ano, aproximadamente cinco milhões de pessoas morrem por doenças relacionadas ao tabaco e a previsão é de que, persistindo o atual modelo de consumo, em 2030, serão oito milhões de mortes ao ano. É mais do que a soma das mortes por alcoolismo, AIDS, acidente de trânsito, homicídio e suicídio juntas ${ }^{(4)}$.

Conforme o Instituto Nacional do Câncer (INCA), o uso combinado do álcool e tabaco vem aumentando globalmente, expondo as pessoas a desenvolverem doenças graves e fatais. Igualmente, essas sustâncias têm alta capacidade de produzir alterações no sistema nervoso central, modificando o comportamento dos indivíduos. Por ter efeito prazeroso, induzem à repetição do seu uso e, assim, à dependência ${ }^{(5)}$.

O consumo de substâncias psicoativas legais e ilegais é mais frequente entre os universitários do que na população em geral, e grande parcela desses estudantes está exposta ao consumo concomitante de múltiplas substâncias psicoativas ${ }^{(2)}$.

Tendo em vista a relevância do tema, este estudo teve como objetivo conhecer a prevalência do consumo de álcool e tabaco entre estudantes de uma universidade do sul do Brasil, dando maior visibilidade a essa problemática, bem como trazendo elementos para tencionar uma reflexão sobre o comportamento da juventude frente às substâncias psicoativas.

\section{MÉTODOS}

O estudo proposto se caracteriza por ser uma pesquisa de caráter exploratório-descritivo, com delineamento de um estudo transversal. A população do estudo foi composta por acadêmicos dos cursos de Medicina, Direito e Engenharia Civil, das respectivas áreas do conhecimento: saúde, humanas e exatas, de uma instituição de ensino superior (IES) na Região Sul do Brasil. Foram convidados a participar $100 \%$ dos acadêmicos matriculados nos referidos cursos, cursando o primeiro, terceiro e/ou último ano de graduação, totalizando 284 participantes de um universo de 303 alunos regularmente matriculados naquele período.

Portanto, como critério de inclusão, os acadêmicos deveriam estar regularmente matriculados na fase do curso em estudo. Quanto aos critérios de exclusão, acadêmicos com idade inferior a 18 anos de idade, ausentes da sala de aula no dia da coleta de dados e/ou preenchimento incorreto do questionário. Não houve recusa de nenhum estudante presente em responder ao 
questionário, porém doze não estavam presentes no dia da coleta de dados e sete não preencheram de forma satisfatória 0 instrumento de pesquisa, sendo excluídos. Computando, assim, uma perda de 6,3\% do total da amostra.

A pesquisa foi realizada por meio de um questionário estruturado de autopreenchimento e sem identificação do acadêmico. As variáveis: idade, sexo, situação conjugal, trabalho, ano de graduação, curso e frequência de uso do álcool e do tabaco (uma ou duas vezes ao ano; mensalmente; semanalmente; diariamente ou quase todos os dias e nunca), foram obtidas por meio de um questionário elaborado com base em outros três instrumentos utilizados em estudos nacionais ${ }^{(6-8)}$. Destaca-se que tais instrumentos foram previamente testados no Brasil pelos autores referendados. Outrossim, as questões eram estruturadas (fechadas) com preenchimento numérico ou múltipla escolha.

Para a coleta de dados, os coordenadores dos cursos participantes do estudo foram contatados para o agendamento do dia e hora mais adequado para a abordagem com os acadêmicos. Identificada a sala, os pesquisadores se apresentaram ao docente responsável pela turma, a quem previamente foram explicados os objetivos e a metodologia da pesquisa, sobre a aplicação do instrumento de pesquisa (obrigatoriamente realizada durante o horário de aula) e quanto tempo iria tomar, aproximadamente 30-45 minutos da aula. Posteriormente, os pesquisadores se apresentaram à turma e explicaram os objetivos, a metodologia, a importância do estudo e fizeram o convite para participarem da pesquisa.

Os questionários preenchidos adequadamente foram digitados em planilhas construídas especificamente para esse estudo no programa Excel. Posteriormente, após revisão da qualidade do preenchimento dos formulários e da planilha, as informações foram transpostas para um banco de dados construídos no programa SPSS versão 20.0.

Em seguida, passaram por uma análise descritiva, com cálculo das frequências absolutas e relativas, e também estatística, em que foi utilizado o teste estatístico qui-quadrado $\left(\chi^{2}\right)$ para verificar associação entre gênero e consumo, assim como a frequência de consumo, de cada substância. A frequência de consumo foi comparada entre cursos com uso de testes KruskalWallis, seguidos de comparações múltiplas não paramétricas de Dunn (quando necessárias entre os sexos) com uso de testes Mann-Whitney. As idades dos alunos foram descritas segundo os cursos, com uso de média e desvio padrão, e comparadas entre os cursos com uso de ANOVA, seguida por comparações múltiplas de Bonferroni. Os testes foram realizados com nível de significância de $5 \%$.

Ressalta-se que o projeto foi aprovado pelo Comitê de Ética em Pesquisa da Universidade Comunitária da Região de Chapecó sob protocolo de pesquisa $n^{\circ}$. 21912. Também foi solicitada para a instituição de ensino superior participante da pesquisa uma declaração de ciência e concordância com a realização do estudo. Aos participantes do estudo foi garantido que não seria divulgado o nome da instituição de ensino superior caso fosse publicada.

\section{RESULTADOS}

Dentre os participantes dessa pesquisa, 34,9\% $(n=99)$ dos acadêmicos eram do curso de Medicina, 27,8\% ( $n=79)$ de Direito e 37\% (n=106) de Engenharia Civil. Segundo os resultados, a média de idade dos acadêmicos era de 23,1 ( \pm 8$)$ anos. Na caracterização da amostra constata-se que 54,6\% (n=155) eram do sexo feminino. Quanto ao estado conjugal, 84,6\% $(n=241)$ são solteiros. Entre os participantes do estudo, 39,3\% $(\mathrm{n}=112)$, além de estudarem, trabalhavam. A amostra foi composta por $32,3 \%(n=92)$ de estudantes do primeiro ano, 36,8\% $(n=104)$ do terceiro e 30,9\% $(n=88)$ do último ano do curso de graduação.

Em relação ao consumo de álcool, $242(85,2 \%)$ dos universitários já consumiram álcool em algum momento na vida. Entre os estudantes do curso de medicina, apenas 6,1\% $(\mathrm{n}=6)$ nunca experimentaram álcool. Quando questionados em relação à frequência com que faziam uso da bebida alcoólica, 38,7\% $(\mathrm{n}=110)$ relataram utilizar álcool semanalmente. Entre os cursos, essa mesma frequência se apresenta da seguinte forma: 34,2\% $(n=27)$ dos acadêmicos de Direito, 34,9\% ( $n=37)$ de Engenharia e 46,5\% (n=46) de Medicina. Quanto ao uso diário de álcool, a prevalência foi de 2,5\% (n=7) entre os acadêmicos. Não houve diferença da frequência de uso entre os cursos $(\mathrm{p}=0,0335)$, conforme a Tabela $\mathrm{I}$.

Tabela I - Frequência do consumo de álcool entre acadêmicos segundo o curso superior de uma universidade do Sul do Brasil, 2012 -2013.

\begin{tabular}{|c|c|c|c|c|c|c|c|c|c|}
\hline \multirow{3}{*}{ Frequência do consumo de álcool } & \multicolumn{6}{|c|}{ Curso } & \multirow{2}{*}{\multicolumn{2}{|c|}{ Total }} & \multirow{3}{*}{ p-valor } \\
\hline & \multicolumn{2}{|c|}{ Medicina } & \multicolumn{2}{|c|}{ Direito } & \multicolumn{2}{|c|}{ Engenharia Civil } & & & \\
\hline & $\mathbf{n}$ & $\%$ & $\mathbf{n}$ & $\%$ & $\mathbf{n}$ & $\%$ & $\mathbf{n}$ & $\%$ & \\
\hline Uma ou duas vezes ao ano & 24 & 24,2 & 18 & 22,8 & 21 & 19,8 & 63 & 22,2 & 0,0335 \\
\hline Mensalmente & 20 & 20,2 & 15 & 19,0 & 27 & 25,5 & 62 & 21,8 & \\
\hline Semanalmente & 46 & 46,5 & 27 & 34,2 & 37 & 34,9 & 110 & 38,7 & \\
\hline Diariamente ou quase todos os dias & 3 & 3,0 & 2 & 2,5 & 2 & 1,9 & 7 & 2,5 & \\
\hline Nunca & 6 & 6,1 & 17 & 21,5 & 19 & 17,9 & 42 & 14,8 & \\
\hline
\end{tabular}


Na relação consumo de álcool e sexo dos universitários, não houve diferença estatística significativa quanto ao fato de já terem consumido uma vez na vida, sendo que $89,1 \%(n=116)$ dos homens e $81,8 \%(n=126)$ das mulheres já experimentaram álcool em algum momento de suas vidas $(\mathrm{p}=0,857)$. Contudo, o consumo semanal do álcool é mais frequente entre o sexo masculino, sendo essa diferença estatisticamente significativa $(p=0,001)$, como se observa na Tabela II.

Tabela II - Descrição da frequência do consumo de álcool segundo o sexo dos acadêmicos de uma universidade do Sul do Brasil, 2012-2013.

\begin{tabular}{|c|c|c|c|c|c|c|c|}
\hline \multirow{3}{*}{ Frequência do consumo de álcool } & \multicolumn{4}{|c|}{ Sexo } & \multirow{2}{*}{\multicolumn{2}{|c|}{ Total }} & \multirow{3}{*}{ p-valor* } \\
\hline & \multicolumn{2}{|c|}{ Masculino } & \multicolumn{2}{|c|}{ Feminino } & & & \\
\hline & $\mathbf{n}$ & $\%$ & $\mathbf{n}$ & $\%$ & $\mathbf{n}$ & $\%$ & \\
\hline Uma ou duas vezes ao ano & 21 & 16,3 & 42 & 27,3 & 63 & 22,3 & 0,001 \\
\hline Mensalmente & 24 & 18,6 & 38 & 24,7 & 62 & 21,9 & \\
\hline Semanalmente & 65 & 49,6 & 45 & 29,2 & 110 & 38,5 & \\
\hline Diariamente ou quase & 6 & 4,7 & 1 & 0,6 & 7 & 2,5 & \\
\hline $\begin{array}{l}\text { todos os dias } \\
\text { Nunca }\end{array}$ & 14 & 10,9 & 28 & 18,2 & 42 & 14,8 & \\
\hline
\end{tabular}

*Resultado do teste qui-quadrado.

Em relação ao uso do tabaco, $18,7 \%(n=53)$ dos acadêmicos relataram que já utilizaram tabaco alguma vez na vida. Os acadêmicos do curso de Direito têm a maior frequência de uso, com uma prevalência de 20,3\% ( $\mathrm{n}=16)$. Já entre os estudantes do curso de Medicina nenhum relatou consumir tabaco diariamente, conforme apresentado na Tabela III.

Tabela III - Frequência do consumo de tabaco entre acadêmicos segundo o curso superior de uma universidade do Sul do Brasil, 2012-2013..

\begin{tabular}{|c|c|c|c|c|c|c|c|c|c|}
\hline \multirow{3}{*}{ Frequência do consumo de tabaco } & \multicolumn{6}{|c|}{ Curso } & \multirow{2}{*}{\multicolumn{2}{|c|}{ Total }} & \multirow{3}{*}{ p-valor* } \\
\hline & \multicolumn{2}{|c|}{ Medicina } & \multicolumn{2}{|c|}{ Direito } & \multicolumn{2}{|c|}{ Engenharia Civil } & & & \\
\hline & $\mathbf{n}$ & $\%$ & $\mathbf{n}$ & $\%$ & n & $\%$ & $\mathbf{n}$ & $\%$ & \\
\hline Uma ou duas vezes ao ano & 10 & 10,1 & 7 & 8,9 & 12 & 11,3 & 29 & 10,2 & 0,816 \\
\hline Mensalmente & 4 & 4,0 & 4 & 5,1 & 1 & 0,9 & 9 & 3,2 & \\
\hline Semanalmente & 3 & 3,0 & 3 & 3,8 & 3 & 2,8 & 9 & 3,2 & \\
\hline Diariamente ou & 0 & 0,0 & 2 & 2,5 & 4 & 3,8 & 6 & 2,1 & \\
\hline $\begin{array}{l}\text { quase todos os dias } \\
\text { Nunca }\end{array}$ & 82 & 82,8 & 63 & 79,7 & 86 & 81,1 & 231 & 81,3 & \\
\hline
\end{tabular}

*Resultado do teste qui-quadrado.

O consumo de tabaco entre os sexos apresenta uma diferença estatisticamente significativa $(\mathrm{p}=0,008)$, evidenciando que os homens consomem mais tabaco que as mulheres, como mostra a Tabela IV.

Tabela IV - Frequência do consumo de tabaco entre acadêmicos segundo o sexo dos universitários de uma universidade do Sul do Brasil, 2012-2013..

\begin{tabular}{|c|c|c|c|c|c|c|c|}
\hline \multirow{3}{*}{ Frequência do consumo de tabaco } & \multicolumn{4}{|c|}{ Sexo } & \multirow{2}{*}{\multicolumn{2}{|c|}{ Total }} & \multirow{3}{*}{ p-valor* } \\
\hline & \multicolumn{2}{|c|}{ Masculino } & \multicolumn{2}{|c|}{ Feminino } & & & \\
\hline & $\mathbf{n}$ & $\%$ & $\mathbf{n}$ & $\%$ & $\mathbf{n}$ & $\%$ & \\
\hline Uma ou duas vezes ao ano & 19 & 14,7 & 10 & 6,5 & 29 & 10,2 & 0,008 \\
\hline Mensalmente & 4 & 3,1 & 5 & 3,2 & 9 & 3,2 & \\
\hline Semanalmente & 6 & 4,7 & 3 & 1,9 & 9 & 3,2 & \\
\hline Diariamente ou quase todos os dias & 4 & 3,1 & 2 & 1,3 & 6 & 2,1 & \\
\hline Nunca & 96 & 74,4 & 135 & 87,0 & 231 & 81,3 & \\
\hline
\end{tabular}

*Resultado do teste qui-quadrado.

\section{DISCUSSÃO}

Entre os acadêmicos entrevistados na presente pesquisa, $85,2 \%$ já consumiram álcool em algum momento na vida. Estudos

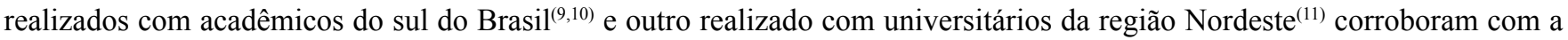
prevalência encontrada: $90,1 \% ; 90,4 \%$; $94 \%$, respectivamente. 
No primeiro levantamento nacional realizado para saber sobre o uso de álcool, tabaco e outras drogas entre universitários das 27 capitais brasileiras, foi possível identificar que cerca de $49 \%$ dos estudantes já experimentaram algum tipo de droga pelo menos uma vez na vida. Comparando com a população geral, os universitários consomem mais drogas que todo o restante da população( ${ }^{(12)}$.

O álcool é a droga lícita de maior prevalência entre estudantes universitários, sendo consumida de forma recreacional, uso de drogas definido como consumo esporádico, embora regular, com a finalidade de diversão e entretenimento. Os estudantes consomem bebidas alcoólicas principalmente em festas e bares, na companhia de amigos e pares ${ }^{(6)}$.

Um estudo sobre o consumo de álcool e interferências na vida acadêmica mostra que 22,65\% dos estudantes fazem o uso do álcool no período das aulas ${ }^{(13)}$. A frequência do consumo de álcool nos cursos acadêmicos da atual pesquisa se apresenta de maneira geral mais prevalente no curso de Medicina. Aproximadamente 34,5\% dos acadêmicos de Direito e de Engenharia Civil consomem álcool semanalmente, enquanto essa prevalência sobe para 46,5\% entre os da Medicina. A rotina estressante dos acadêmicos de Medicina tem sido o principal fator responsável pela dependência destes a qualquer droga, sendo o álcool a mais utilizada entre eles, o que altera sua percepção diante dos casos de abuso da bebida alcoólica, visto que esses estudantes tendem a ser mais tolerantes e se consideram menos vulneráveis ao álcool ${ }^{(14)}$. O consumo de álcool entre estudantes dessa área é preocupante, pois se espera que tenham um maior conhecimento a respeito das implicações de tais substâncias na saúde de cada indivíduo ${ }^{(15)}$.

Na relação consumo de álcool e sexo dos acadêmicos, não há uma diferença, entretanto, quando analisada a frequência do uso, os acadêmicos do sexo masculino consomem álcool numa assiduidade semanal maior que as do sexo feminino. De acordo com outras pesquisas realizadas, as diferenças frente à frequência e ao consumo de substâncias psicoativas foram observados quando comparados os sexos, já que os universitários homens consomem substâncias ilícitas com maior frequência que as universitárias e apresentam uma prevalência e uma frequência de uso de bebidas alcoólicas quase três vezes maior do que as mulheres ${ }^{(10,12,15)}$. A maioria das mulheres bebe ocasionalmente, enquanto a maioria dos homens ingere álcool de uma a duas vezes por semana, assim sendo, o padrão de uso nocivo e de dependência do sexo masculino, quando comparado ao feminino, é bem maior, indicando que homens ingerem álcool de forma mais prejudicial à saúde do que as mulheres ${ }^{(10,16-18)}$.

Quanto ao consumo de tabaco, houve uma prevalência de 18,9\%. Em semelhança metodológica com a presente pesquisa, outro estudo, realizado entre universitários do sul do Brasil, com uma amostra dividida em dois grandes grupos, área da saúde e demais cursos, a prevalência de tabagismo entre os acadêmicos foi de $8,9 \%$, sendo 4,7\% tabagistas ativos e 4,2\% fumantes ocasionais; e 2,6\% declararam-se ex-fumantes ${ }^{(19)}$. Em outro estudo, com o objetivo de verificar a prevalência ao tabagismo entre os acadêmicos de Medicina, observou-se que 16,5\% dos acadêmicos eram fumantes ativos, 5,4\% diários e 11,1\% ocasionais ${ }^{(20)}$.

Quanto ao sexo, a presente pesquisa também evidenciou que os acadêmicos do sexo masculino têm uma prevalência maior no consumo de tabaco, e a frequência de uso semanal predomina entre eles. Outros estudos apontam que a frequência e quantidade entre mulheres acadêmicas é menor que entre os rapazes ${ }^{(19-21)}$. Apesar da prevalência do uso ser menor entre as mulheres, a OMS, em 2010, elegeu para o "Dia Mundial sem Tabaco" o tema "Gênero e tabaco com ênfase na propaganda voltada para a mulher", com o intuito de promover políticas de combate à epidemia de tabagismo entre as mulheres devido as peculiaridades dos seus efeitos na saúde da mulher ${ }^{(22)}$.

O estudo apontou uma alta prevalência do consumo de álcool entre os universitários. Esse consumo elevado pode estar relacionado aos ambientes da universidade, já que neste meio os jovens frequentam festas em que geralmente há disponibilidade de bebidas alcoólicas, expondo-os também ao consumo de outras substâncias psicoativas ${ }^{(23)}$. Dentre os fatores que influenciam e até mesmo propiciam o consumo de drogas, ilícitas ou não, está o acesso à universidade, em que o jovem se afasta/liberta do seio familiar - muitas vezes indo para centros distantes da sua origem - e vivencia festas em que as drogas por vezes podem ser usadas para favorecer a integração e os relacionamentos interpessoais ${ }^{(11)}$. Além das festas universitárias, também são fatores que propiciam o consumo dessas substâncias as comemorações pós-provas e também como recompensa por encerrar um dia cansativo e estressante de estudos, além das propagandas existentes na mídia, que estimulam o consumo de álcool e tabaco por estas serem drogas lícitas ${ }^{(17)}$.

O consumo de drogas entre os universitários preocupa, pois ocasiona impactos econômicos e sociais, aumentando os índices de violência e abandono dos estudos, necessitando que cada vez mais sejam repensadas as ações e políticas para essa problemática. Dessa maneira, o ambiente universitário deve ser otimizado para promover discussões, pois existe a liberdade para o debate dos mais variados temas, incluindo a criação de espaços e ambientes permanentes para esta discussão(24).

Considerando as limitações deste estudo quantitativo, que buscou identificar o padrão de consumo de álcool e tabaco, tornam-se necessárias investigações mais abrangentes e de abordagens qualitativas, numa perspectiva de compreender as representações e significados do consumo dessas drogas entre os acadêmicos.

\section{CONCLUSÃO}

O presente estudo conclui que a prevalência do uso de álcool entre os universitários é alta, uma vez que um terço dos acadêmicos o consome semanalmente. A frequência de consumo é maior entre acadêmicos do sexo masculino. Quanto ao 
consumo de tabaco, também observou uma maior frequência de uso entre os acadêmicos homens. Os achados apontam paridades no consumo de álcool e tabaco entre os cursos pesquisados. Estes resultados, referentes à realidade do uso de álcool e tabaco entre universitários dos cursos de Medicina, Direito e Engenharia civil, de uma universidade do sul do Brasil, não se difere das demais regiões do Brasil.

\section{REFERÊNCIAS}

1. Lorant V, Nicaise P, Soto VE, D'hoore W. Alcohol drinking among college students: college responsibility for personal troubles. BMC Public Health. 2013;13(615):1-9.

2. Barreto ML, Almeida N Filho. Epidemiologia e saúde: fundamentos, métodos e aplicações. Rio de Janeiro: Guanabara Koogan; 2012.

3. World Health Organization. Global status reporto $\mathrm{n}$ alcohol and health [Internet]. Geneva: WHO; 2014 [acesso em 2015 Abr 11]. Disponível em: http://apps.who.int/iris/bitstream/10665/112736/1/9789240692763_eng.pdf?ua=1

4. World Health Organization. WHO report on the global tobacco epidemic 2008: the MPOWER packaged. Geneva: WHO; 2008 [acesso em 2015 Abr 11]. Disponível em: http://www.who.int/tobacco/mpower/2008/en

5. Instituto Nacional de Câncer - INCA. Consumo de bebidas alcoólicas [Internet]. Rio de Janeiro: INCA [acesso em 2015 Abr 18]. Disponível em: http://www1.inca.gov.br/conteudo_view.asp?ID=14

6. Oliveira HP Júnior, Brands B, Cunningham J, Strike C, Wright MGM. Percepção dos estudantes universitários sobre o consumo de drogas entre seus pares no ABC Paulista, São Paulo, Brasil. Rev Latinoam Enferm. 2009;17(Nespe):871-7.

7. Wagner GA. Álcool e drogas: terceira pesquisa sobre atitudes e uso entre alunos da Universidade de São Paulo - Campus São Paulo (Cidade Universitária, Complexo da Saúde e Faculdade de Direito). São Paulo: Universidade de São Paulo; 2011.

8. Ministério da Saúde (BR), Secretaria de Atenção à Saúde. Política Nacional de Promoção da Saúde [Internet]. $3^{\text {a }}$ ed. Brasília: Ministério da Saúde; 2010 [acesso em 2015 Abr 18]. Disponível em: http://bvsms.saude.gov.br/bvs/publicacoes/ politica_nacional_promocao_saude_3ed.pdf

9. Natividade JC, Aguirre AR, Bizarro L, Hutz CS. Fatores de personalidade como preditores do consumo de álcool por estudantes universitários. Cad Saúde Pública. 2012;28(6):1091-1100.

10. Pedrosa AAS, Camacho LAB, Passos SRL, Oliveira RVC. Consumo de álcool entre estudantes universitários. Cad Saúde Pública. 2011;27(8):1611-21.

11. Picolotto E, Libardoni LFC, Migott AMB, Geib LTC. Prevalência e fatores associados com o consumo de substâncias psicoativas por acadêmicos de enfermagem da Universidade de Passo Fundo. Ciênc Saúde Coletiva. 2010;15(3):645-54.

12. Brasil. Presidência da República. Secretaria Nacional de Políticas sobre Drogas. I levantamento nacional sobre o uso de álcool, tabaco e outras drogas entre universitários das 27 capitais brasileiras [acesso em 2014 Out 21]. Disponível em: http://www.grea.org.br/I_levantamento/I_levantamento nacional.pdf

13. Pereira MO, Cardoso LCS, Souza LMCG, Sampaio VM, Oliveira MAF. O consumo de álcool e outras drogas entre estudantes universitários: interferências na vida acadêmica. SMAD Rev Eletrônica Saúde Mental Alcool Drog [Internet]. 2013 [acesso em 2015 Abr 18];9(3):105-10. Disponível em: http://dx.doi.org/10.11606/issn.1806-6976.v9i3p105-110

14. Mesquita EM, Nunes AJ, Cohen C. Avaliação das atitudes dos estudantes de medicina frente ao abuso de drogas por colegas do meio acadêmico. Rev Psiquiatr Clín (São Paulo). 2008;35(1):8-12.

15. Pedrosa AAS, Camacho LAB, Passos SRL, Oliveira RVC. Consumo de álcool entre estudantes universitários. Cad Saúde Pública. 2011;27(8):1611-21.

16. Paduani GF, Barbosa GA, Morais JCR, Pereira JCP, Almeida MF, Prado MM, et al. Consumo de álcool e fumo entre os estudantes da Faculdade de Medicina da Universidade Federal de Uberlândia. Rev Bras Educ Méd. 2008;32(1):66-74.

17. Carneiro ALM, Rodrigues SB, Gherardi-Donato ECS, Guimarães EAA, Oliveira VC. Padrão do uso de álcool entre estudantes universitários da área da saúde. Rev Enferm Cent.-Oeste Min. 2014;4(1):940-50.

18. Fachini A, Furtado EF. Uso de álcool e expectativas do beber entre universitários: uma análise das diferenças entre os sexos. Psicol Teor Pesqui. 2013;29(4):421-8. 
19. Rosa MI, Caciatori JFF, Panatto APR, Silva BR, Pandini JC, et al. Uso de tabaco e fatores associados entre alunos de uma universidade de Criciúma (SC). Cad Saúde Colet (Rio J). 2014; 22(1):25-31.

20. Stramari LM, Kurtz M, Silva LCC. Prevalência e fatores associados ao tabagismo em estudantes de medicina de uma universidade em Passo Fundo (RS). J Bras Pneumol. 2009;35(5):442-8.

21. Magliari RT, Pagliusi AL, Previero BM, Menezes FR, Feldman A, Novo NF. Prevalência de tabagismo em estudantes de faculdade de medicina. Rev Med. 2008;87(4):264-71.

22. Lombardi SEM, Prado GF, Santos UP, Fernandes FLA. O tabagismo e a mulher: riscos, impactos e desafios. J Bras Pneumol. 2011;37(1):118-128.

23. Pillon SC, O’Brien B, Chavez KAP. A relação entre o uso de drogas e comportamentos de risco entre universitários brasileiros. Rev Latinoam Enferm. 2005;13(Nesp 2):1169-76.

24. Portugal FB, Siqueira MM. Fatores associados ao uso de substâncias psicoativas entre universitários de pedagogia da universidade federal do Espírito Santo. Cad Saúde Colet (Rio J). 2011;19(3):348-55.

\section{Endereço para correspondência}

Lucimare Ferraz

Av. Senador Attílio Fontana, 591-E

Bairro: Efapi

CEP 89809-000 - Chapecó- SC - Brasil

E-mail: lferraz@unochapeco.edu.br 